\title{
Automated detection of satellite contamination in incoherent scatter radar spectra
}

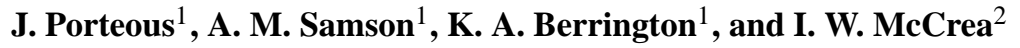 \\ ${ }^{1}$ School of Science and Mathematics, Sheffield Hallam University, Sheffield, S1 1WB, UK \\ ${ }^{2}$ Space Science Department, Rutherford Appleton Laboratory, Chilton, Oxon, OX11 0QX, UK
}

Received: 4 July 2002 - Revised: 4 December 2002 - Accepted: 17 January 2003

\begin{abstract}
Anomalous ion line spectra have been identified in many experiments. Such spectra are defined as deviations from the standard symmetric "double-humped" spectra derived from incoherent scatter radar echoes from the upper atmosphere. Some anomalous spectra - where there are sharp enhancements of power over restricted height ranges - have been attributed to satellite contamination in the beam path. Here we outline a method for detecting such contamination, and review in detail a few cases where the method enables the identification of anomalous spectra as satellite echoes, subsequently ascribed to specific orbital objects. The methods used here to identify such satellites provide a useful way of distinguishing anomalous spectra due to satellites from those of geophysical origin. Analysis of EISCAT Svalbard Radar data reveals that an average of 8 satellites per hour are found to cross the beam. Based on a relatively small sample of the data set, it appears that at least half of the occurrences of anomalous spectra are caused by satellite contamination rather than being of geophysical origin.
\end{abstract}

Key words. Ionosphere (auroral ionosphere, instruments and techniques) - Radio Science (signal processing)

\section{Introduction}

Incoherent scatter radars have been used to study the Earth's ionosphere since their introduction in 1959. The European Incoherent Scatter radar (EISCAT) in Northern Scandinavia is positioned in the auroral zone and is reviewed in Risbeth and Williams (1985). By contrast the EISCAT Svalbard radar (ESR) is positioned further north in the polar cap region $\left(74^{\circ}\right.$ magnetic latitude). It first started its operations in 1996 and a detailed introduction to the system can be found in Wannberg et al. (1997). The information obtained from these radars is contained both in the spectral shape of the scattered signal and in the power returned. The majority of the ion line spec-

Correspondence to: J. Porteous

(J.Porteous@shu.ac.uk) tra are of the standard symmetric "double-humped" type as expected. There has been much interest in those spectra with enhanced ion-acoustic shoulders since the time when they were first identified in incoherent scatter spectra (Foster et al., 1988) using data from the Millstone Hill radar in Westford, Massachusetts. Other authors (Rietveld et al., 1991; Collis et al., 1991; Forme, 1995) have reported occurrences of anomalous spectra in the data from the Troms $\varnothing$ radar in Norway.

Many theories have been proposed to account for the occurrences of such spectra. The earliest of these suggests that they may be due to current-driven instabilities, but in this situation the field-aligned current densities would need to be unusually high (Collis et al., 1991; Rietveld et al., 1991). A second possibility is that they may be due to the parametric decay of Langmuir waves (Forme, 1995), whilst a third suggestion is that they are caused by ion-ion two-stream instabilities (Wahlund et al., 1992).

Many examples of naturally enhanced ion acoustic spectra have been reported in the ESR data. Recently, Buchert et al. (1999) suggest that the ion-acoustic fluctuation level seems to depend quite strongly on the wave number, $k$. In their review paper, Sedgemore-Schulthess et al. (1999) discuss examples of enhanced spectra in the context of smallscale aurora, transient field-aligned currents and time varying magnetic reconnection. For a detailed review of naturallyenhanced ion-acoustic spectra, see Sedgemore-Schulthess and St-Maurice (2001).

Given the large size of incoherent scatter data sets, the only acceptable method of identifying such spectra is to use an automated program to search the data. However, as the paper by Rietveld et al. (1996) indicates, there are many other causes of spectral anomalies, the most common of which is satellite contamination. The identification of anomalous echoes of geophysical interest would be greatly facilitated if a reliable algorithm could be found to identify and reject the satellite contamination. As a result, we have attempted to find a way of automatically checking the data for anomalous spectra and isolating those cases which are clearly caused by satellites. 

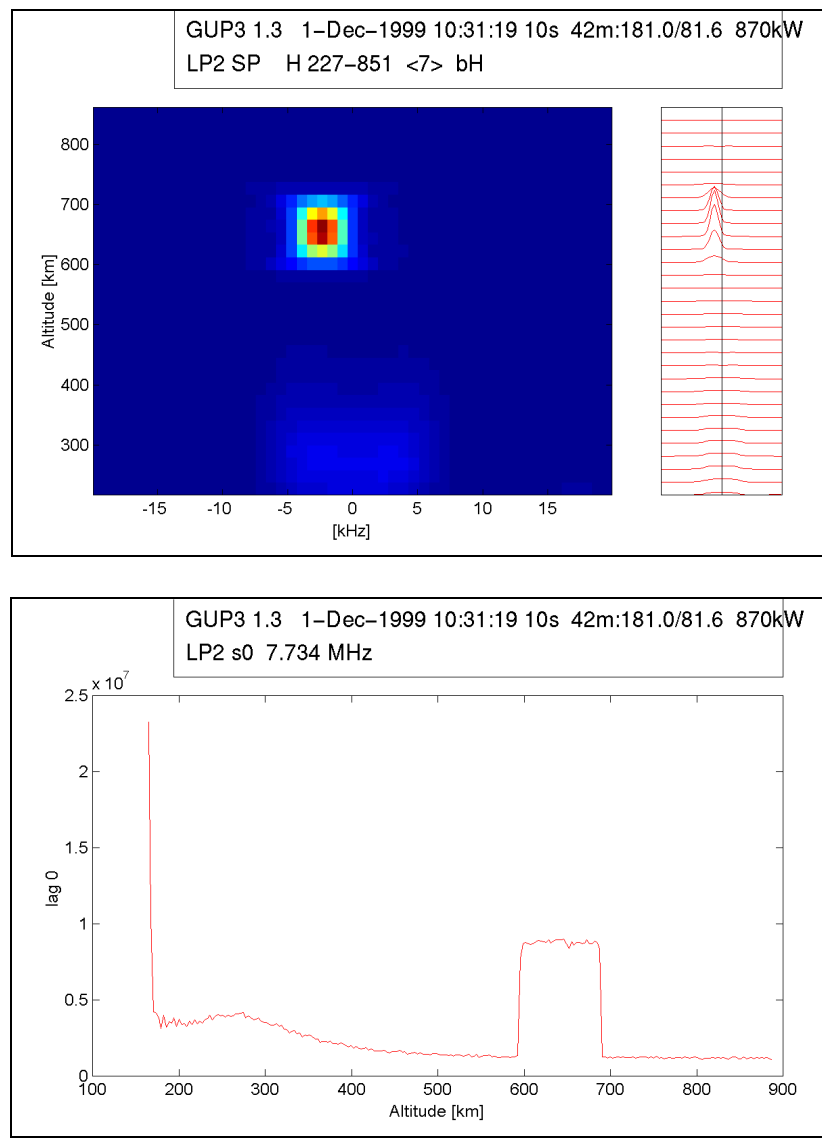

Fig. 1. Results showing (a) spectra, and (b) power profile for LP2 data at 10:31:19 UT, 1 December 1999, using EISCAT rtg3 program when a probable satellite was seen in the beam.

\section{Identifying enhanced spectra}

The data used in this study come from the EISCAT Svalbard Radar (ESR), and are measured using the GUP3 experiment. This experiment makes use of both plain, long pulses and alternating codes, which are binary codes based on phasecoded long pulses. The use of two different modulations with overlapping range coverage is important, as it allows the presence of anomalies to be confirmed by comparing the data from the two pulse schemes. The experiment makes use of two long pulse channels, LP1 and LP2, and two channels for alternating code data, $\mathrm{AC} 1$ and $\mathrm{AC} 2$. In this study, we discuss results from the comparison of the data from LP2 and $\mathrm{AC} 2$, although $\mathrm{LP} 1$ and $\mathrm{AC} 1$ were also checked to ensure that the results are consistent. The range covered by the LP2 data is $229.5-859.5 \mathrm{~km}$, whilst that covered by the AC2 data is $228-732 \mathrm{~km}$. Hence, the LP and AC data overlap within a region whose range extent is about $500 \mathrm{~km}$. Within this region, full measurements of the shape of the backscattered spectrum can be obtained from both schemes, along with "power profiles", representing the variation in the backscattered power as a function of range.

A simple method is employed to search for anomalous ion-
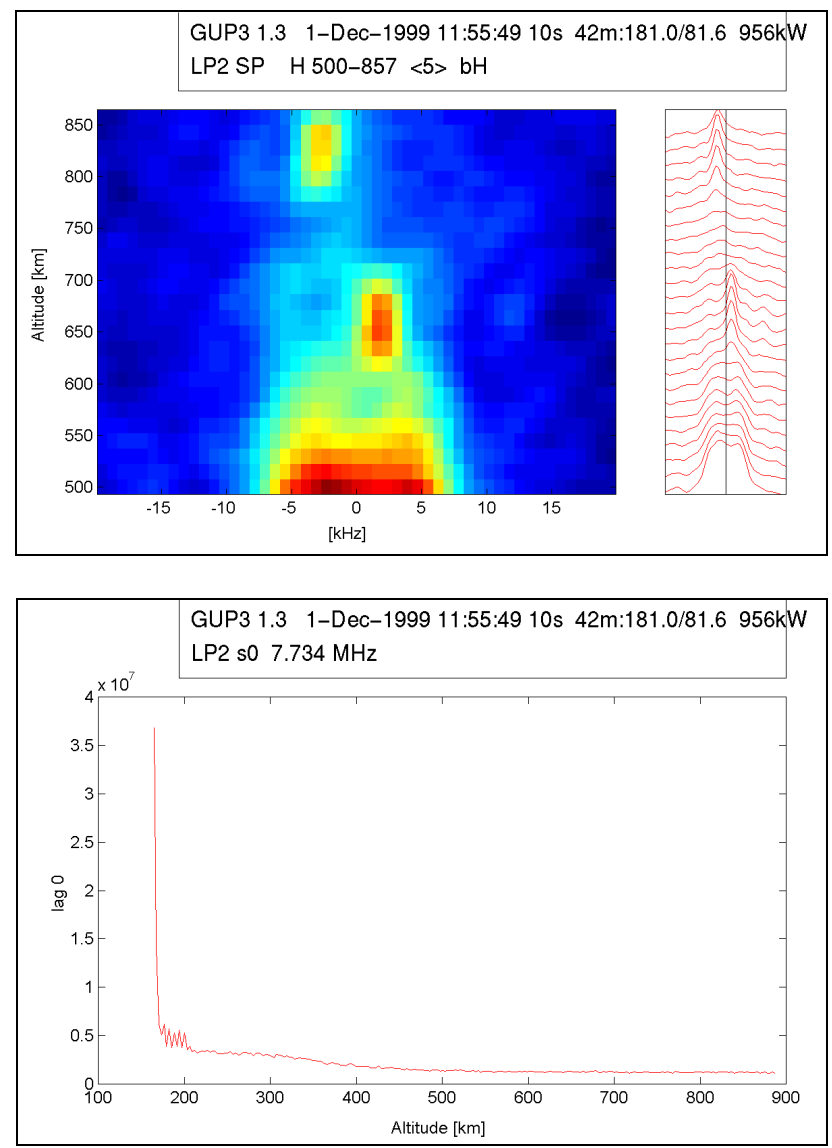

Fig. 2. Results showing (a) spectra, and (b) power profile for LP2 data at 11:55:49 UT, 1 December 1999, using EISCAT rtg3 program when enhanced spectra probably due to geophysical causes were seen in the beam.

line spectra, based on the asymmetry between the upshifted and downshifted ion-acoustic peaks. First, we obtain the frequency at which the spectrum attains its maximum value. We call this the primary peak. Finding a secondary peak, if one exists, is more difficult. Instead, we restrict attention to the data more than $2.5 \mathrm{kHz}$ from the primary peak and find the frequency at which the maximum occurs in this remaining data set. On most occasions, it can be verified that these two frequencies represent the peaks of the ion-acoustic lines. However, when the secondary peak is much lower than the primary one, the program will not identify the secondary peak. Instead, it identifies a point on a shoulder of the primary peak. When the ratio of the heights of these two points is less than some threshold value, the details are stored in a file for later examination. After making several observations, the arbitrary number 0.8 is chosen as the threshold value. This is seen to ensure that those situations of interest to us are identified along with a small number of spurious cases which subsequently can be eliminated by a manual check.

In this paper, we present a typical example of the GUP3 data, from 1 December 1999. The data set consists of 
1011 ten-second dumps recorded between 10:11:30 UT and 13:00:00 UT. The automated routine described above is used to examine the data from the LP2 long-pulse channel. The times at which anomalous spectra are identified by the program are recorded for subsequent manual examination. In some cases, it transpires that the spectra identified as being anomalous contain essentially no backscattered signal and any such noisy spectra are eliminated. This leaves a number of spectra where the program suggests the presence of a satellite. Typical enhanced spectra from such a case are shown in Fig. 1a. In particular, the power profiles for these times are checked. When the returned signal is thought to be the result of a reflection from a satellite or item of space debris, the power profile measured on the LP2 channel shows a sudden sharp increase, followed by a similarly sudden sharp decrease approximately $100 \mathrm{~km}$ further along the beam, as shown in Fig. 1b. The spacing between these steps in the power is related to the range ambiguity of the sounding pulse, so a satellite large enough to cause a significant change in the returned power is detected in several range gates. This explains why the power increase is visible over a $100 \mathrm{~km}$ range. Sometimes the object detected is at a sufficiently high altitude for only the increase in power, but not the subsequent decrease, to be within the range coverage of the recorded data. These situations are classified as possible satellites.

In contrast to this example, there are times when there are enhanced spectra in the data but the power profile shows no significant change, as shown in Fig. 2. The spectra obtained at these times can, therefore, be the result of a geophysical phenomenon. In a situation where the enhancements in one ion line switch to the other line (Sedgemore-Schulthess et al., 1999), the enhancements are thought to be short-lived as are those for satellites. However, any increase in power which occurs is likely to be gradual rather than sudden, so it takes several range gates for the full increase to show in such geophysical situations. The example shown in Fig. 2 needs further consideration if it is to be identified definitely, either as a geophysical phenomenon or as one or more satellites passing through the beam.

A detailed study of the variations between adjacent points in the power profile reveals that a few very large power variations are seen, which are clearly attributable to satellites. Excluding these, the remaining variations show a continuous distribution, rather than being sharply divided between large and small changes. The distribution is shown in Fig. 3. In order to accommodate the full range of power ratio variations on the same graph, the natural logarithm of the power ratio variation has been plotted.

Considering the histogram in Fig. 3, the cases at the lefthand extreme of the $x$-axis are highly likely to be due to geophysical conditions, whilst those at the right-hand extreme are highly likely to be due to satellites. This leaves a large number of intermediate points, many of which could also be due to satellites, either of varying cross section, or passing through the side lobes rather than the main beam.

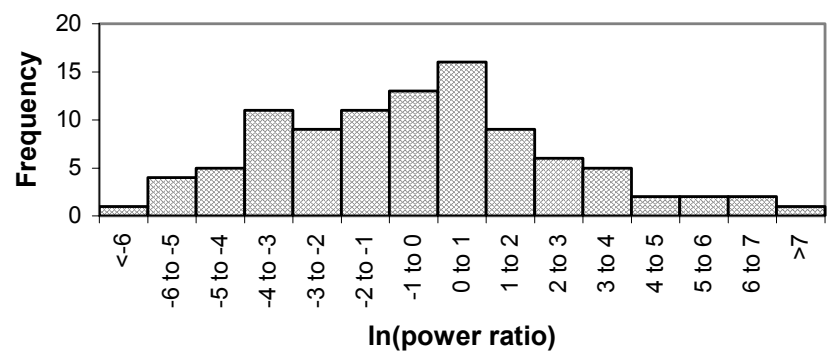

Fig. 3. Histogram showing frequency of occurrence of $1 n$ of power ratios obtained when enhanced spectra occurred in the 1 and 2 December 1999 ESR, GUP3 data.

\section{Long pulse and alternating code comparisons}

To progress further, a comparison is made between the long pulse (LP2) results and those from the alternating code (AC2) data. The range extent of the LP2 long pulse data is 229.5$859.5 \mathrm{~km}$, whilst for the AC2 data it is only $228-732 \mathrm{~km}$. As a consequence, only a subset of the anomalous spectra identified in the LP2 data can be checked using the AC2 data.

By examining the power profile from the $\mathrm{AC} 2$ data at the relevant times, similar characteristics can be seen, as shown in Fig. 4. When the enhanced spectra are probably due to a satellite passing through the beam, the $\mathrm{AC} 2$ power profile shows a steep pointed peak; otherwise, there is no significant change. The smaller extent of the peak in the power profile response arises because the range resolution is better for the alternating code data than it is for the long pulse data. At these times the peak of the AC power is confined to a very narrow range and is within the region in which the step occurs in the LP data. Thus, good agreement is shown between the two pulse schemes. In addition, the use of the AC data helps to clarify a number of cases where the LP data alone does not provide an unambiguous identification of a satellite.

In order to classify the data, we use a threshold ratio for the LP power increases between adjacent range gates above which the enhancement is probably caused by a satellite. Below this ratio geophysical causes are more likely, but there is a possibility that the anomally is caused by very small items of space debris or by a satellite being detected in the far side lobes of the radar beam. For the data we are considering here we suggest that an appropriate threshold to use is 0.2 . This represents a $20 \%$ increase in the returned power. Examples which are close to the threshold are checked manually.

We now consider ways of calibrating such identifications, to verify that satellites or their debris have indeed passed through the beam.

\section{Checking for satellites}

The monitoring of space debris, and the determination of its orbital parameters is an important activity for the space industry. Many such objects are routinely monitored and their positions can subsequently be reconstructed. An element file 

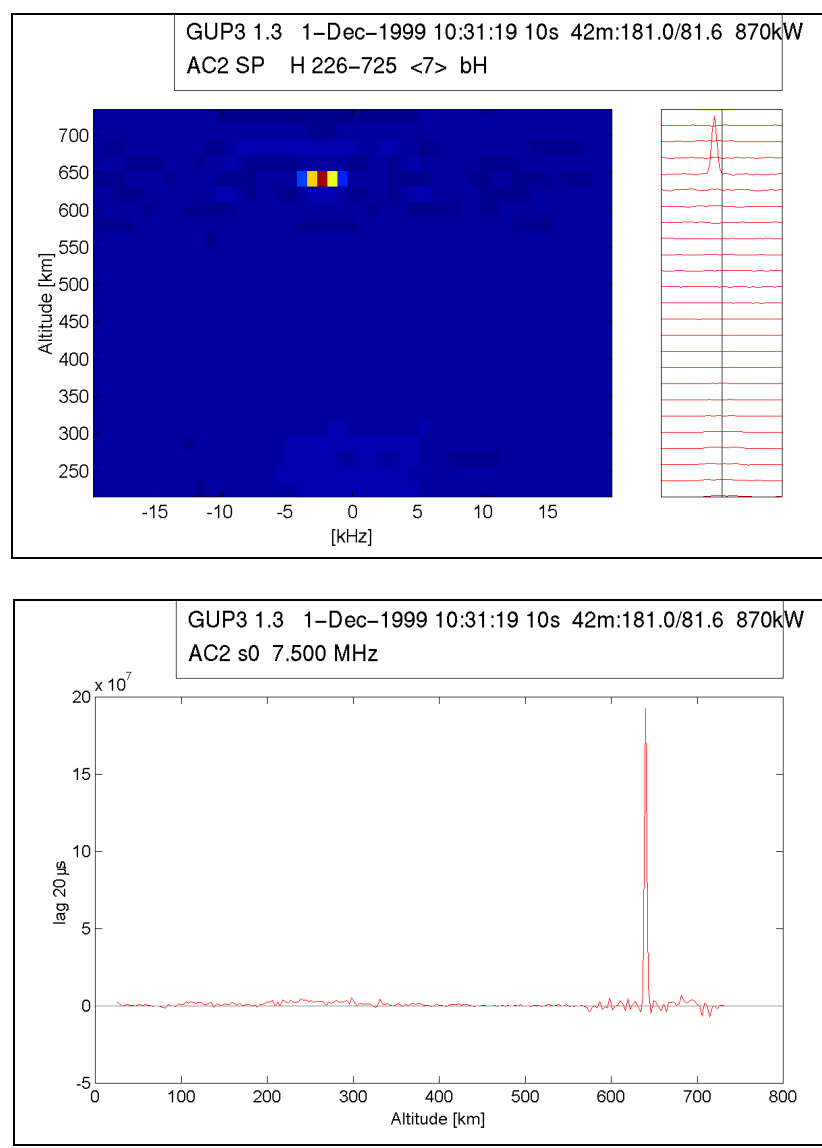

Fig. 4. Results showing (a) spectra, and (b) power profile for AC2 data at 10:31:19 UT, 1 December 1999, using EISCAT rtg3 program when a probable satellite was seen in the beam.

for satellites and other space debris with NORAD catalogue numbers, (University of Arizona, 2002), updated on 2 December 1999, is used to check for the occurrence of satellites in the ESR radar beam at various times on 1 December 1999.

At 10:31:13 UT an object with catalogue number 24134$94029 \mathrm{GH}$ is found to have had an azimuth of $179.88^{\circ}$, an elevation of $81.63^{\circ}$ and a range of $652 \mathrm{~km}$ relative to the EISCAT Svalbard Radar, as shown in Fig. 5.

The radar data indicates that at 10:31:19 UT (i.e. the 10-s dump starting at 10:31:10 UT) there was a probable satellite visible in the beam. The range of the observed anomalous echo is in good agreement with the predicted position of the object.

Using spherical trigonometry, the distance of the object from the centre of the radar beam can be calculated. The radar beam had an elevation of $81.6^{\circ}$ and an azimuth of $181.0^{\circ}$. Hence, the identified object was $1.9 \mathrm{~km}$ from the centre of the beam. This is well within the limits of the expected lateral extent $(\sim 6.5 \mathrm{~km})$ of the main beam at a range of $\sim 650 \mathrm{~km}$.

This example, together with five other times at which the prediction software successfully confirms the presence of a satellite or item of space debris within the range of the beam, are shown in Table 1. The examples identified at 10:31:13 UT and 12:04:10 UT are likely to have been detected within the main beam, whilst the rest are more likely to have been detected in the side lobes. At each of these times the power profiles for both the LP2 and the AC2 returned signal show the typical characteristics described earlier, suggesting that the sounding pulses were being reflected from a hard target. The object detected at 11:36:35 UT is likely to have been quite large, hence, the high value obtained for the power ratio.

At 12:07:32 UT a satellite is noticed to be $3.210^{\circ}$ from the centre of the radar beam and at $834 \mathrm{~km}$ along its range. This is too great a range to enable it to be visible in the AC2 data. However, the LP2 data does show enhanced spectra around this range, as well as the expected increase in power. The increase in power, though significant, is not as great as in the earlier examples. This is possibly a result of the larger distance from the beam centre, suggesting that the satellite passed through the side lobes rather than the main beam. A further satellite at 12:08:21 UT is identified to be $2.849^{\circ}$ from the centre of the beam, but at a range of $871 \mathrm{~km}$. This is not detected in either the AC2 nor the LP2 data. Additionally, at 11:55:30 UT a satellite $6.319^{\circ}$ from the centre of the radar beam and at a range of $815 \mathrm{~km}$ is not detected in the LP2 data.

\section{Conclusions}

Over 1000 ten-second dumps of raw data from the EISCAT Svalbard Radar have been studied, in order to find a method for identifying likely causes of enhanced spectra. It is suspected that many of the cases found are due to satellites or space debris passing through the main beam or the side lobes. An automated routine has been developed and used to identify those times at which enhanced spectra occur.

It is shown that examining the variation of power with range gives a good indication of whether the spectra are caused by satellites or are possibly of a geophysical nature. In the ESR GUP3 experiment, which contains both long pulse and alternating code data, enhancements in the power profiles of both modulations are noted when satellites appear. A threshold increase of $20 \%$ in the returned power is used to help divide the cases into two classes. Situations in which the returned power shows an increase of $20 \%$ or more are classified as being due to satellites or space debris. Those which show a smaller increase in power need further investigation to see if they have a geophysical cause. On this basis, it is possible to use an automated program to distinguish between such cases. A small number of noisy spectra are identified by the program and have to be eliminated.

In the period 10:11:30 UT to 13:00:00 UT on 1 December 1999 the numbers of satellites and possibly naturally enhanced spectra occurring in the region covered by the ESR radar beam are as given in Table 2. It can be seen that there are, on average, about eight satellites per hour passing through the radar beam. 


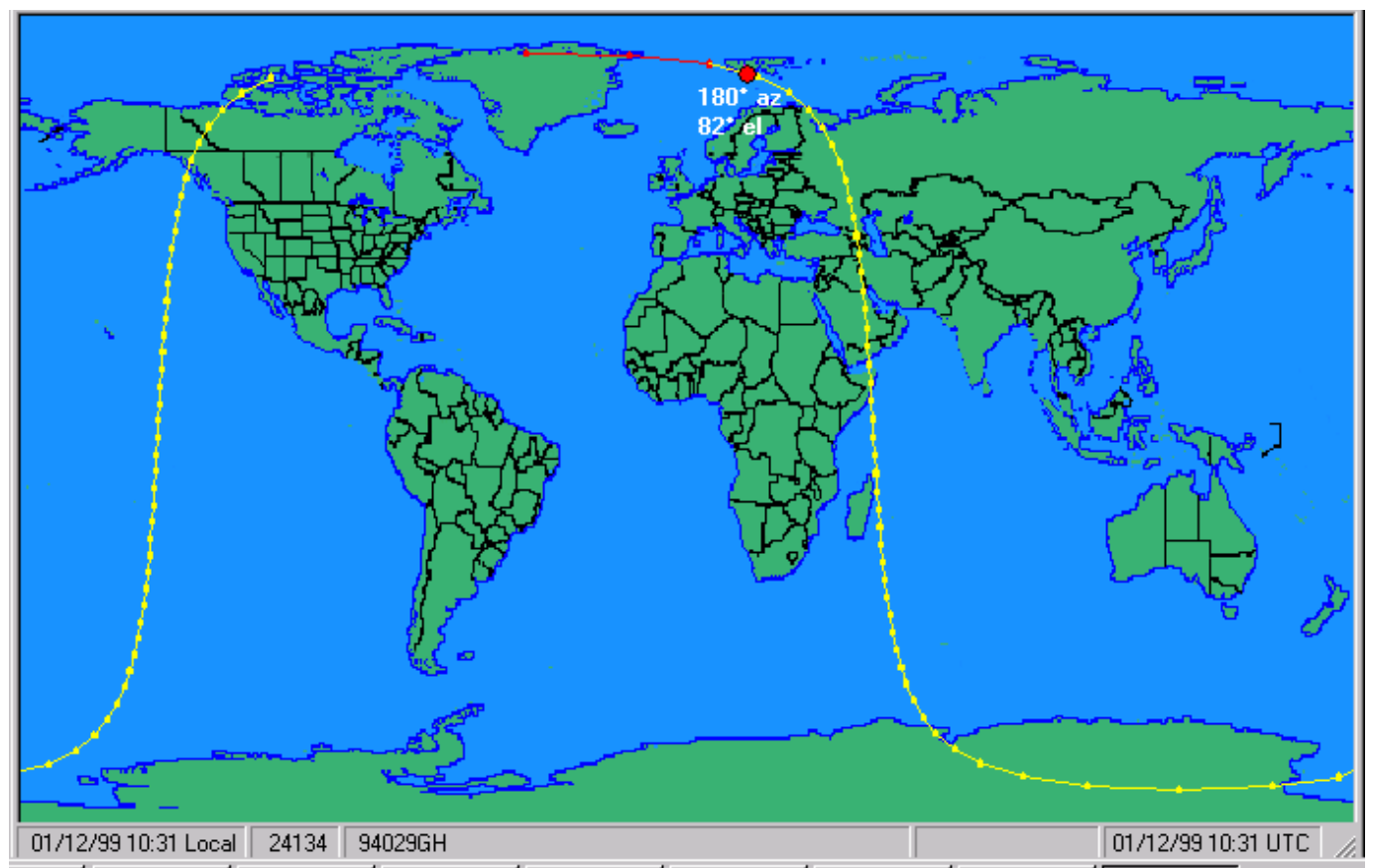

Fig. 5. Path of space debris, NORAD number 24134-94029GH, showing its position relative to the radar at ESR at 10:31:13 UT on 1 December 1999.

Table 1. A sample of times at which a satellite or item of space debris appeared within the ESR beam on 1 December 1999

\begin{tabular}{ccccc}
\hline $\begin{array}{c}\text { Time (UT) } \\
\text { on 1 Dec 1999 }\end{array}$ & NORAD Cat. No. & $\begin{array}{c}\text { Angle from centre } \\
\text { of radar beam }\left(^{\circ}\right)\end{array}$ & Range (km) & Power Ratio \\
\hline $10: 30: 36$ & $24149-94029 \mathrm{GY}$ & 1.400 & 629 & 1.796 \\
$10: 31: 13$ & $24134-94029 \mathrm{GH}$ & 0.166 & 652 & 4.788 \\
$11: 36: 35$ & $25919-99051 \mathrm{~A}$ & 0.310 & 706 & 733.497 \\
$12: 04: 10$ & $12760-81053 \mathrm{DD}$ & 0.195 & 762 & 4.467 \\
$12: 06: 32$ & $25941-99057 \mathrm{~B}$ & 0.278 & 771 & 0.462 \\
$12: 16: 27$ & $20466-90010 \mathrm{~B}$ & 1.394 & 641 & 2.906 \\
\hline
\end{tabular}

Table 2. The number of satellites and possibly naturally enhanced spectra found in the region covered by the ESR beam for 10:11:30 UT to 13:00:00 UT on 1 December 1999

\begin{tabular}{ccc}
\hline Range $(\mathrm{km})$ & $\begin{array}{c}\text { Number of } \\
\text { satellites }\end{array}$ & $\begin{array}{c}\text { Number of possibly } \\
\text { naturally enhanced spectra }\end{array}$ \\
\hline$<600$ & 2 & 1 \\
$600-750$ & 7 & 5 \\
$>750$ & 15 & 10 \\
\hline
\end{tabular}

Satellites which are at too great a distance, such as that predicted at 12:08:21 UT with a range of $871 \mathrm{~km}$, are not detected by the radar. Similarly, those at too large an angle from the beam centre, such as that predicted at 11:55:30 UT at an angular distance of $6.319^{\circ}$, are not detected. This gives some insight into the area within which the beam at Svalbard could detect satellite contamination. Further work needs to be carried out to provide greater refinement.

The routine has been tested using a further $6 \mathrm{~h}$ of data on 2 December 1999 and almost $16 \mathrm{~h}$ of data on 3 December 1999 and similar results have been obtained. Since there are a large number of satellites, or items of space debris, orbiting the Earth and, therefore, likely to pass through the radar beam, care must be taken to distinguish between the spectra caused by such phenomena and those caused by geophysical conditions. This applies not only to the ESR data, but to any radar data.

Further studies still need to be performed at the times of those anomalous echoes, not automatically rejected as being due to satellite contamination, to determine whether or not these are genuinely of a geophysical nature. Since the occurrences of enhanced spectra due to geophysical conditions are small, automated search routines of the type described here provide the scope for extracting such data from very large extended data sets. This method can assist in the investigation of naturally enhanced spectra and their causes. 
Acknowledgements. We are indebted to the director and staff of EISCAT for opening the facility and supplying the data. EISCAT is an international facility supported by the national science councils of Finland, France, Germany, Japan, Norway, Sweden, and the United Kingdom. We thank Mike Rietveld, Russell Eberst and Alan Pickup for their help in obtaining the satellite data and directing us to appropriate tracking programs. Our thanks are also due to Rick von Glahn for allowing us to use results from the Element Manager for Windows program. The authors have greatly appreciated the helpful comments and suggestions made by the referee. The satellite tracking software and catalogue files used in this study were obtained from the following wedsites: http://www.eoss.org/elementmanager/index.html and ftp://ftp.seds.org/pub/sat/satelem/el-1999/

Topical Editor M. Lester thanks F. Sedgemore-Schulthess for his help in evaluating this paper.

\section{References}

Buchert, S. C., van Eyken, A. P., Ogawa, T., and Watnabe, S.: Naturally enhanced ion-acoustic lines seen with the EISCAT Svalbard Radar, Adv. Space. Res., 23, 1699-1704, 1999.

Collis, P. N., Häggström, I., Kaila, K., and Rietveld, M. T.: EISCAT radar observations of enhanced incoherent scatter spectra; their relation to red aurora and field-aligned currents, Geophys. Res. Lett., 18, 1031, 1991.

Forme, F. R. E: Parametric decay of beam-driven Langmuir wave and enhanced ion-acoustic fluctuations in the ionosphere: a weak turbulence approach, Ann. Geophysicae, 17, 1172, 1995.
Foster, J. C., del Pozo, C., and Groves, K.: Radar observations of the onset of current driven instabilities in the topside ionosphere, Geophys. Res. Lett., 15, 160, 1988.

Rietveld, M. T., Collis, P. N., van Eyken, A. P., and Lovhaug, U. P.: Coherent echoes during EISCAT UHF Common Programmes, J. Atmos. and Terr. Phys., 58, 161-174, 1996.

Rietveld, M. T., Collis, P. N., and St-Maurice, J.-P.: Naturally enhanced ion acoustic waves in the auroral ionosphere observed with the EISCAT 933-MHz Radar, J. Geophys. Res., 96, 19291 , 1991.

Risbeth, H. and Williams, P. J. S.: The EISCAT ionospheric radar: The system and its early results, Q. J. R. Astr. Soc., 26, 478-512, 1985.

Sedgemore-Schulthess, F., Lockwood, M., Trondsen, T. S., Lanchester, B. S., Rees, M. H., Lorentzen, D. A., and Moen, J.: Coherent ESR spectra from the dayside cusp/cleft and their implications for transient field-aligned currents, J. Geophys. Res., 104, 24 613-24 624, 1999.

Sedgemore-Schulthess, F. and St-Maurice, J.-P.: Enhanced ionacoustic spectra and their interpretation, Surv. Geophys., 22, 1, 55-92, 2001.

Wahlund, J.-E., Forme, F. R. E., Opgenoorth, H. J., and Persson, M. A. L.: Scattering of electromagnetic waves from a plasma: Enhanced ion-acoustic fluctuations due to ion-ion two-stream instabilities, Geophys. Res. Lett., 19, 1919-1922, 1992.

Wannberg, G., Wolf, I., Vanhainen, L. G., Koseniemi, K., Röttger, J., Postila, M., Jacobsen, R., Stenberg, A., Larsen, R., Eliasen, S., Heck, S., and Huuskonen, A.: The EISCAT Svalbard Radar: A case study in modern incoherent scatter radar system design, Radio Sci., 32, 2283-2307, 1997. 